\title{
A Study of Heart Rate and Brain System Complexity and Their Interaction in Sleep-Deprived Subjects
}

\author{
AK Kokonozi, EM Michail, IC Chouvarda, NM Maglaveras \\ Aristotle University of Thessaloniki, Thessaloniki, Greece
}

\begin{abstract}
In this work we investigate the synchronization of the dynamic behaviour of heart rate (ECG) and brain (EEG) signals using sample entropy as a measure of complexity. EEG and ECG recordings were collected during experiment with sleep-deprived subjects exposed to real field driving conditions. The degree to which brain and heart complexity loose complexity in a synchronous manner, indicating a possible interaction between the two systems is investigated. Preliminary results obtained from the examination of four subjects show the existence of a weak-to-intermediate cross-correlation between these pairs of biological oscillators. Furthermore, the frequency content in both heart rate and brain signals was calculated via power spectrum analysis and the association of synchronisation patterns with prevalent frequencies in the two systems was investigated.
\end{abstract}

\section{Introduction}

One of the main reasons for many fatal road accidents is the fatigue of sleep-derived drivers. Drowsiness leads to lengthen reaction time, decreases vigilance and attention and slows information processing [1]. Heart Rate Variability (HRV) and electroencephalogram (EEG) are two physiological factors that co-vary with drowsiness levels [2-6] addressing an underlying common central mechanism. EEG is a brain activity measure, able to track variations in alertness [7] while drowsiness estimation can be achieved with the use only of central and posterior channels [8]. Spectral analysis of HRV indicates drivers fatigue by increased High Frequencies (HF) and decreased Low Frequencies (LF) and LF/HF [9].

The association of EEG and ECG recordings using frequency analysis [10] demonstrated an inverse correlation between delta band in EEG and LF, LF/HF from HRV analysis, suggesting that sympathetic nervous activities became decreased with sleep deepening and increased with sleep lightening.

This study aims to determine possible correlations between the dynamic behaviour of heart rate (ECG) and brain (EEG) signals in order to explore the interaction between these two systems for sleep-derived drivers in real field conditions. The complexity measure employed is the sample entropy which is a probabilistic estimate of the pointwise match, within a tolerance, between the two signals. Synchronisation patterns are also investigated between $\mathrm{HF}$ and theta waves $(4-8 \mathrm{~Hz})$, which is the predominant frequency during the transition state between wakefulness and sleep [11].

\section{Methods}

Twenty one subjects (20 male and 1 female) with a mean age of 26.5 years were participated in the real field driving experiment, which was performed in CERTH, Thessaloniki, Greece, from 6 June to 27July 2007, using the CERTH experiment car. The participants remained awake at least $24 \mathrm{~h}$ before the experiment under supervision.

Electrodes attached to the subject, ensured the acquisition of EEG and ECG signals through an ambulatory monitoring system, supported by a battery. An experienced driving instructor was seated at the codriver's seat. A technician monitoring the functioning of the recording equipment was in the back seat and a medical doctor monitoring EEG and ECG data, was next to him.

An electro-cap connected to the recording device used for the EEG data collection from positions Fp1, Fp2, C3, $\mathrm{C} 4, \mathrm{P} 3, \mathrm{P} 4, \mathrm{O} 1$, and $\mathrm{O} 2$. For the needs of ECG recording, electrons were positioned on the sternum and the fifth intercostal space on the left side of the body.

The sampling rate used was $200 \mathrm{~Hz}$, with amplitude range of $\pm 20 \mu \mathrm{V}$ for EEG signals. The monitoring system hardware filters band passed data in the range: $0.5 \mathrm{~Hz}$ to $70 \mathrm{~Hz}$ for EEG, with a notch filter at $50 \mathrm{~Hz}$ power supply component.

EEG data were first filtered using a $3^{\text {rd }}$ order Butterworth filter (band pass range: $0.5-0.45 \mathrm{~Hz}$ ). For the ECG data, initially the mean ECG value was subtracted from the original channel data. The resulted data were band passed with a $6^{\text {th }}$ order Butterworth filter (band pass range: $2-70 \mathrm{~Hz}$ ). R-wave intervals (RRI) were extracted [12], [13] and artifacts were corrected 
[14], before calculating Heart Rate [15].

Recordings analyzed for this study satisfied some conditions: I) the drivers managed to accomplish around one hour driving task, II) driving errors occurred during the measurements, III) not too many driving error occurred during the experiment, causing difficulties to the study of the features under consideration around the events.

The chosen subjects were: Subject 1, Subject 2, Subject 3, Subject 4. Karolinska Sleepiness scale: 7-8, 7, 7 , respectively.

EEG recordings were divided into 2 minutes windows. For each channel, the energy in bands delta $(0.5-4 \mathrm{~Hz})$, theta $(4-8 \mathrm{~Hz})$, alpha $(8-12 \mathrm{~Hz})$, beta $(12-30 \mathrm{~Hz})$, and gamma $(30-40 \mathrm{~Hz})$ was calculated.

RR intervals (RRI) were extracted from the ECG recordings, while power spectrum components (LF and HF) of the Heart Rate were calculated in 4 min window with $75 \%$ overlap. Sample Entropy of the exported RR intervals was also calculated in segments of 4 minutes with step 30 seconds.

\section{Results}

The drivers participated in this experiments started to feel drowsiness around twenty minutes after they started driving. In order to assess that transition from awake to drowsiness in the association of the recorded signals, we calculated the correlation between EEG and ECG sample entropy and between HF (from ECG) and Theta band (from EEG) not only for all the signals duration, but also for those parts that correspond to the first twenty minutes of driving and the rest time of the driving experiment.

The figures below show the results for the Subjects under consideration.

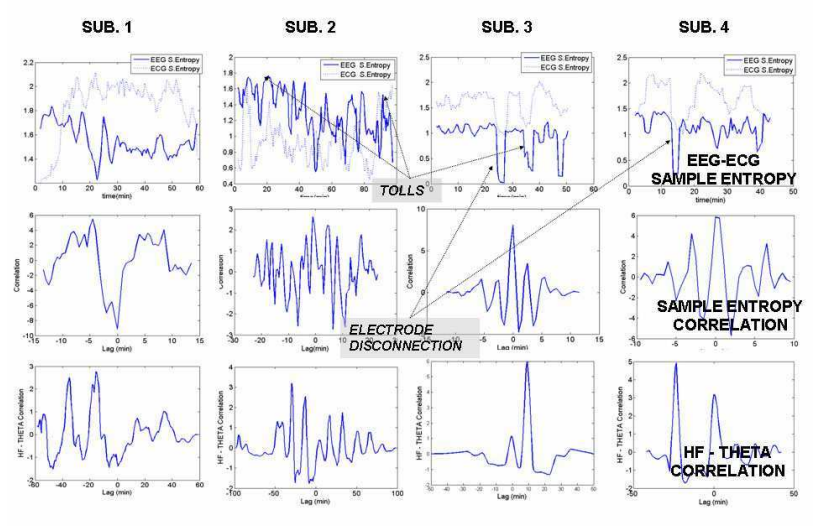

Figure 1. EEG and ECG Sample Entropy for each Subject in the first line of the Figure. Correlation between EEG and ECG Sample Entropy (plots in the second line) and HF - Theta band, for each Subject (plots in the third line).
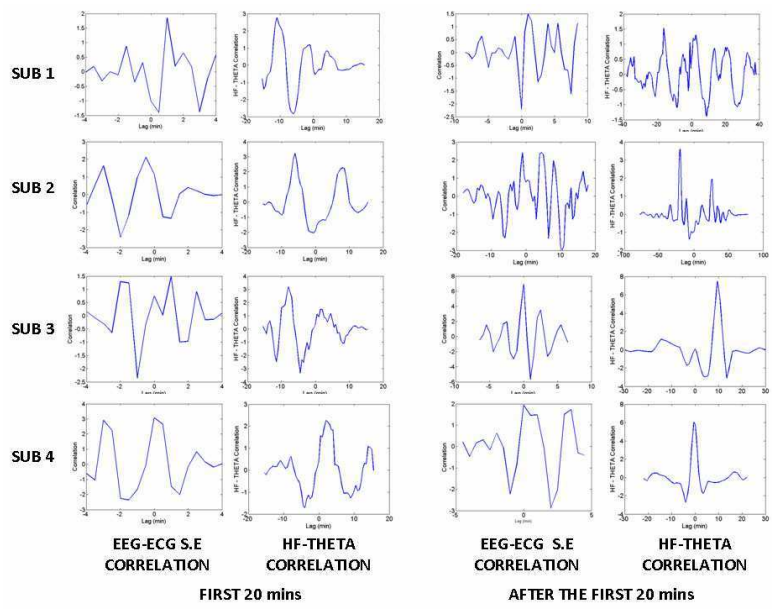

Figure 2. Correlation between EEG-ECG sample entropy and HF - Theta waves fore each Subject, during the first twenty minutes of driving and for the rest time of the experiment in real field conditions.

In the above figures we can see that the synchronization between EEG and ECG sample entropy can be either positive or negative with lags also positive (ECG ahead) or negative (EEG ahead) and close to zero. The negative correlation or correlation with negative lags is more evidenced in the first twenty minutes of driving, while after that time more fluctuations in this synchronization can be observed. This decrease of synchronization might be a light indication of the driver drowsiness.

The conclusions for the HF and Theta band are similar but with higher correlation lags. For the first twenty minutes of driving Theta waves seem to be forward, while this head can be switched to HF for the rest part of the driving task.

The synchronization between the above signals is positive in cases like tolls and electrode disconnection. Again the lag for HF and Theta is higher.

The correlation coefficient for EEG and ECG sample entropy in a sliding window of 5 minutes was also calculated. During the reported driving errors (vertical lines in the plots, Figure 3) lower values of the correlation coefficient were observed, indicating loss of the signals synchronization during the events. 


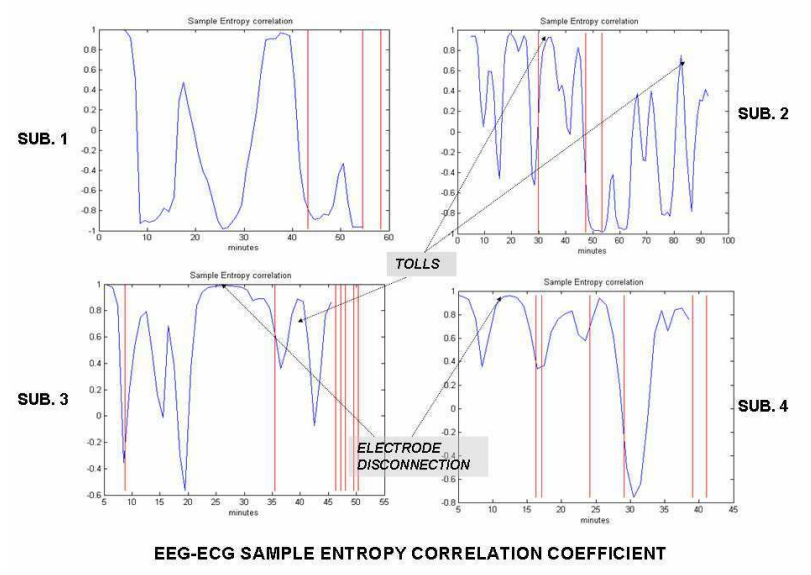

Figure 3. Correlation coefficient for EEG-ECG sample entropy for each Subject.

\section{Discussion and conclusions}

This study shows interesting preliminary results for the synchronization of heart and brain signals during real field driving conditions. A slight interaction between these systems can be observed, which varies during the driving task, depending on the drivers fatigue and special events (e.g. tolls).

In order to assess and determine the correlation of EEG and ECG signals, we need to further investigate their dynamic behaviour under different driving conditions (more Subjects of different age and health status in different drowsiness level and driving conditions).

\section{Acknowledgements}

Special thanks to Prof. Klonowski, Group of Biosignal Analysis Fundamentals, Polish Academy of Sciences Medical, for kindly making available to the SENSATION consortium the Higuchi fractal dimension calculation method.

This work was partly supported by the EU-IST-2003507231 project SENSATION.

\section{References}

[1] Nikhil RP, Chien-yao C, Li-wei K, Chih-feng C, Tzyyping J, Chin-teng L et al. EEG-based Subject-and Sessionindependent Drowsiness Detection: An Unsupervised Approach. J EURASP of Advances in Signal Processing 2008.

[2] Huang RS, Kuo CJ, Tsai LL, Chen OTC. EEG Pattern Recognition Arousal States Detection and Classification. Proc.IEEE Conf. Neural Netw 1996 ; 641-6.

[3] Vuckovic A, Radivojevic V, Chen AC, Popovic D. Automatic Recognition of Alertness and Drowsiness from
EEG by an Artificial Neural Network. Med. Eng. Phys $2002 ; 349-360$.

[4] Roberts S, Rezek I, Everson R, Stone H, Wilson S, Alford C. Automated Assessment of Vigilance using Committees of Radial Basis Function Analysers. Proc. IEEE Sci. Meas. Technol 2000; 333-8.

[5] Khalifa KB, Bedoui MH, Raytchev R, Dogui M. A Portable Device for Alertness Detection. Int. IEEE EMBS Special Topic Confe. Microtechnolog. Med. Biol 2000 ; 584-6.

[6] Wilson BJ, Bracewell TD. Alertness Monitor using Neural Networks for EEG Analysis. IEEE Signal Process. Soc. Workshop on Neural Netw. Signal Process 2000; 814-820.

[7] Papadelis C, Kourtidou-papadeli C, Bamidis PD, Chouvarda I, Koufogiannis D, Bekiaris E, Maglaberas N. Indicators of Sleepiness in an Ambulatory EEG study of Night Driving. EMBS Proceedings 2006 ; 6201-4.

[8] Chin-teng L, Ruei-chengwu, Tzyy-ping J, Sheng- Fu L, Teng-yi $\mathrm{H}$ et al. Applied signal processing. J EURASIP 2005; 19:3165-3174.

[9] Liang VC, Yuan J, Sun DC, Lin MH. Variation in Physiological Parameters Before and After an Indoor Simulated Driving Task: Effect of Exercise Break. International Conference on Gerontic Technology and Service Management (ICGTSM) 2007 ;

[10] Ako MT, Kawara T, Uchida S, Miyazaki S, Nishihara K, Mukai J, Hirao K, Ako J, Okubo Y, et al. Correlation between electroencephalography and heart rate variability during sleep. J Psychiatry and Clinical Neurosciences 2003; 57:59- 65 .

[11] NeuroDevelopment.Neuro Science Information. [homepage on the Internet]. 2006 [cited 2008 Jul 15]. Available from: http://www.neurodevelopment.com/en/brainwaves.htm

[12] Alfonso, Tompkins VX, Nguyen WJ, Shen Luo Tq et al. ECG beat detection using filter banks. J IEEE transactions on biomedical engineering 1999; 46:192 - 202.

[13] Analysis of heart rate variability in the presence of ectopic beats using the heart timing signal. J IEEE Transactions on biomedical engineering 2003; 50.

[14] Berger RD . An efficient algorithm for apectral analysis of heart rate variability. J IEEE Transactions Biomed. Eng. 1986; 33:900-904.

[15] Taskforce of the European Society for Cardiology and the North American Society of Pacing and Electrophysiology . Heart rate variability: standards of measurement, physiological interpretation and clinical use. J European Heart Journal 1996; 17:354-381.

\section{Address for correspondence}

Nicos Maglaveras.

Lab of Medical Informatics, Medical School, Aristotle University of Thessaloniki, 54124, Greece.

nicmag@med.auth.gr 